\title{
NO PLACE TO HIDE: IS THE CIGARETTE A POTENTIAL CESSATION TOOL?
}

Louise M. Hassan, Lancaster University, U.K. Edward Shiu, Bangor University, U.K.

\begin{abstract}
The present paper makes two contributions to the literature, firstly by investigating whether or not three potential changes to the appearance of cigarettes would lead to positive benefits in increasing intentions to quit smoking. Secondly, by assessing which message would potentially work best on the cigarette. A scenario based field experiment with 125 current smokers was conducted. Smokers were randomly assigned into one of four conditions. Thirty seven smokers saw a cigarette which was completely black. A black cigarette was intended to convey a message of tar and disease as black is often associated with death and fear. However black can also be associated with elegance, authority and power. Thirty four smokers saw a cigarette which was printed with a message about the number of minutes that each inhalation of tobacco smoke would reduce the life expectancy of the smoker. The final experimental condition contained thirty smokers who saw a cigarette with the chemicals contained in cigarettes printed on it. Twenty four smokers were in a control condition. Smokers were not given the opportunity to handle the cigarettes but saw a photograph of the cigarette in an ash tray. Prior to the smokers seeing the photograph of the cigarette intention to quit smoking was solicited. After viewing the photograph the smokers were asked questions relating to the persuasiveness of the messages contained on the cigarette and intention to quit smoking.

It was expected that a significant increase in intention to quit post against pre exposure would occur in the experimental conditions but not in the control condition. Examining the change pre and post exposure revealed a significant main effect on intention to quit $(F=13.56 ; P<0.001)$ and a significant interaction between exposure and condition $(F=6.17 ; P<0.01)$ on intention to quit. Significant differences were found for both the minute $(t=-.3 .81 ; P<0.01)$ and chemicals $(t=-2.25 ; P<$ 0.05 ) conditions, with increases in intention to quit smoking found post exposure for both conditions (minute: $M_{\text {pre }}=3.26$ vs. $M_{\text {post }}=3.94$; chemicals: $M_{\text {pre }}=3.83$ vs. $\left.M_{\text {post }}=4.07\right)$. Results revealed that only persuasiveness $\left(\chi^{2}=14.45 ; P<0.001\right.$; from analyses including persuasiveness, refutations, believability, and perceived value) and the interaction between persuasiveness and condition $\left(\chi^{2}=4.03 ; P<0.05\right)$ were significant. Respondents in the minute condition exhibit a stronger effect of persuasion on intention than those in the chemicals condition. These results suggest that changing the appearance of the cigarette by including the display of health information may result in higher intentions to quit smoking. Furthermore those strategies which make concrete the temporal effect of smoking, particularly relating to minutes of life lost, work best in this situation.
\end{abstract}

References Available Upon Request 